\title{
Optimized radial and angular positions in Monte Carlo modeling
}

\author{
Lihong Wang and Steven L. Jacques \\ Laser Biology Research Laboratory, The University of Texas M.D. Anderson Cancer Center, \\ 1515 Holcombe Blvd., Houston, Texas 77030
}

(Received 3 January 1994; accepted for publication 17 March 1994)

In Monte Carlo simulations of light transport in tissues, a grid system is set up to score physical quantities. This study of cylindrically symmetrical problems found that the optimized radial and angular positions for the averaged physical quantities in each grid element are off-center. The error of the extrapolated physical quantities at the light-incidence point using the centered radial positions is up to $14.3 \%$.

Since Wilson and Adam ${ }^{1}$ first introduced Monte Carlo simulations into the field of laser-tissue interactions, the method has been widely used to simulate photon transport in tissues. ${ }^{2-11}$ In the simulations, laser light is injected into the tissue (turbid medium) and is traced statistically. A grid system is set up to score physical quantities, including reflectance, transmittance, or absorption. In cylindrically symmetrical problems, e.g., when a laser beam is perpendicular to a multilayered tissue slab, a grid system is usually established to score light in the radial $(r)$ domain, where the radius is the distance between the observation point and the laserincidence point on the tissue slab. Usually, the center of each $r$-grid element is used for the average physical quantity in the grid element. This communication first proves that the optimized-r positions are off-center in each $r$-grid element and then briefly discusses the angle $(\alpha)$, depth $(z)$, and time $(t)$ domains.

The grid separation in the $r$ domain is denoted by $\Delta r$, and the total number of grid elements is $N$. The index to each grid element is denoted by $n$, where $0 \leqslant n \leqslant N-1$. The center of each grid element is denoted by $r_{n}$,

$$
r_{n}=(n+0.5) \Delta r \text {. }
$$

Monte Carlo simulations approximate the average of the physical quantity $Y(r)$ in each grid element, where $Y(r)$ can be diffuse reflectance, diffuse transmittance, or internal fluence at a particular $z$ value. Although $Y(r)$ is not analytically known, which is why we perform numerical simulations, the average of $Y(r)$ in the $n$ th-grid element can be expressed mathematically by

$$
\langle Y(r)\rangle=\frac{1}{2 \pi r_{n} \Delta r} \int_{r_{n}-\Delta r / 2}^{r_{n}+\Delta r / 2} Y(r) 2 \pi r d r
$$

where the term $2 \pi r_{n} \Delta r$ is the area of the grid element in the form of a circle for $n=0$, or of an annulus for $n>0$.

If $Y(r)$ in each grid element is differentiable and is approximated linearly, there exists a best point $r_{b}$ to satisfy

$$
\langle Y(r)\rangle=Y\left(r_{b}\right)
$$

Therefore, the simulated average should be assigned to $r_{b}$.

$Y(r)$ in the $n$ th-grid element can be approximated by a Taylor series expanded to the first order about $r_{b}$,

$$
Y(r) \approx Y\left(r_{b}\right)+\left(r-r_{b}\right) Y^{\prime}\left(r_{b}\right) .
$$

Substituting Eq. (4) into Eq. (2), i.e.,

$$
\begin{aligned}
\langle Y(r)\rangle= & \frac{1}{2 \pi r_{n} \Delta r} \int_{r_{n}-\Delta r / 2}^{r_{n}+\Delta r / 2}\left[Y\left(r_{b}\right)+\left(r-r_{b}\right) Y^{\prime}\left(r_{b}\right)\right] \\
& \times 2 \pi r d r
\end{aligned}
$$

and completing the integration yields

$$
\langle Y(r)\rangle=Y\left(r_{b}\right)+Y^{\prime}\left(r_{b}\right)\left[r_{n}+\frac{(\Delta r)^{2}}{12 r_{n}}-r_{b}\right] .
$$

It we set the term $\left[r_{n}+(\Delta r)^{2} / 12 r_{n}-r_{b}\right]$ in Eq. (6) to 0 and solve for $r_{b}$, Eq. (6) becomes Eq. (3) and we thus obtain

$$
r_{b}=r_{n}+\frac{\Delta r}{12 r_{n}} \Delta r
$$

or

$$
r_{b}=\left[(n+0.5)+\frac{1}{12(n+0.5)}\right] \Delta r .
$$

Therefore, the optimized-r positions for $n=0,1,2,3,4, \ldots$ are $(2 / 3) \Delta r, 1.556 \Delta r, 2.533 \Delta r, 3.524 \Delta r, 4.519 \Delta r, \ldots$, correspondingly. The optimized-r position deviates from the center of each grid element as a result of the $2 \pi r$ factor in the integration in Eq. (2). The smaller the index to the grid box, the larger is the deviation. As the index $n$ becomes large, the optimized- $r$ position approaches the grid-element center. For $n=0$, the difference of the center from the optimized- $r$ position is $\Delta r / 6$ or $25 \%$.

Linear interpolation is usually used to estimate $Y(r)$ in between grid points and extrapolation is used to estimate $Y(r=0) . Y(r)$ that is estimated using the centers of grid elements will be different from the more accurate $Y(r)$ that is estimated using the optimized- $r$ positions, and the difference is the greatest for $Y(r=0)$, as illustrated in Fig. 1. The average simulation results for $Y(r)$ in grid elements $n=0$ and 1 are denoted by $Y_{0}$ and $Y_{1}$, respectively. If we use the centers of the grid elements, $Y(r=0)$ is

$$
\begin{aligned}
Y_{c}(0) & =Y_{0}-\left(Y_{0}-Y_{1}\right)(\Delta r / 2) /(\Delta r / 2-3 \Delta r / 2) \\
& =Y_{0}+\left(Y_{0}-Y_{1}\right) / 2 .
\end{aligned}
$$

If we use the optimized-r positions, $Y(r=0)$ is

$$
\begin{aligned}
Y_{b}(0) & =Y_{0}-\left(Y_{0}-Y_{1}\right)(2 \Delta r / 3) /(2 \Delta r / 3-14 \Delta r / 9) \\
& =Y_{0}+3\left(Y_{0}-Y_{1}\right) / 4 .
\end{aligned}
$$

The relative error between $Y_{b}(0)$ and $Y_{c}(0)$ is 


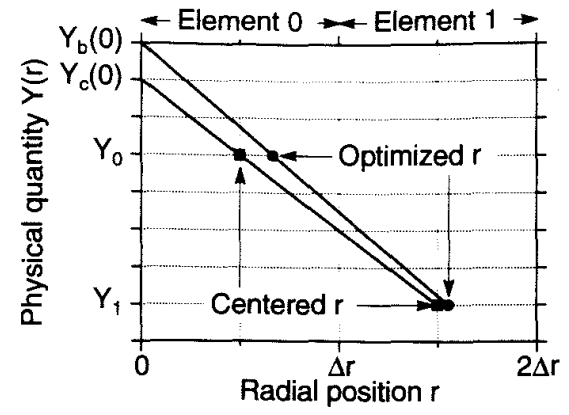

FIG. 1. An illustration estimating the physical quantity $Y(r)$ at $r=0 . Y_{0}$ and $Y_{1}$ denote the simulated average physical quantity in elements $n=0$ and 1 , respectively. The grid separation is $\Delta r . Y_{b}(0)$ and $Y_{c}(0)$ are the extrapolated estimations of $Y(r=0)$ using the optimized- and centered-r positions, respectively.

$$
\left[Y_{b}(0)-Y_{c}(0)\right] / Y_{b}(0)=\left(1-Y_{1} / Y_{0}\right) /\left(7-3 Y_{1} / Y_{0}\right) \leqslant 1 / 7
$$

The relative error varies with the ratio between $Y_{1}$ and $Y_{0}$ and approaches the maximum $1 / 7(14.3 \%)$, when $Y_{1} / Y_{0}$ approaches 0 . For example, if $Y_{1} / Y_{0}$ is 0.1 , the relative error is about $13 \%$.

Using the centers of grid elements also leads to errors when convolution is used to calculate the light distribution for laser beams of a finite width. An impulse response for an infinitely narrow beam that is perpendicularly incident upon a multilayered tissue slab can be first calculated using Monte Carlo simulations. The response of the beam with a finite width can then be computed through convolution of the impulse response over the beam profile. ${ }^{2}$ (Both our Monte Carlo simulation and convolution programs are in the public domain and can be obtained by contacting the authors of this paper.) Figure 2 shows the impulse diffuse reflectance for an infinitely narrow laser beam of $1 \mathrm{~J}$ energy and the diffuse reflectance of a flat laser beam of $0.01 \mathrm{~cm}$ radius and $1 \mathrm{~J}$

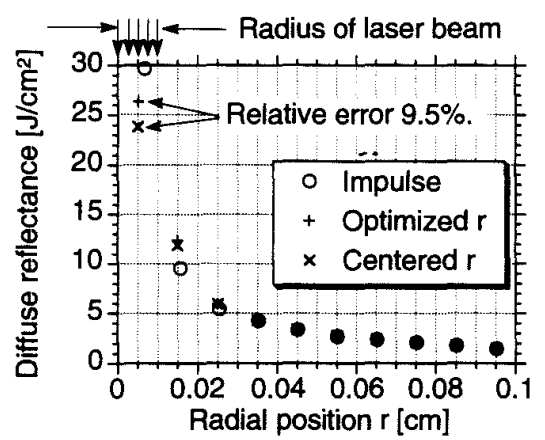

FIG. 2. Diffuse reflectance of a laser beam that is perpendicularly incident upon an infinitely thick tissue slab in air. The optical properties of the tissue are refractive index $=1.37$, absorption coefficient $=1.0 \mathrm{~cm}^{-1}$, scattering coefficient $=100.0 \mathrm{~cm}^{-1}$, and anisotropy $=0.9$. The circles show the Monte Carlo-simulated impulse response of an infinitely narrow laser beam of $1 \mathrm{~J}$ energy at optimized-r positions, where the grid separation in the radial direction is $0.01 \mathrm{~cm}$ and the number of grid elements is 100 during the Monte Carlo simulation. The pluses and crosses show the diffuse reflectances in response to a flat laser beam of $1 \mathrm{~J}$ energy and $0.01 \mathrm{~cm}$ radius convolved using the optimized- and centered-r positions, respectively. energy that is perpendicularly incident upon an infinitely thick tissue slab. The convolved diffuse reflectances computed using the optimized- and centered-r positions are different near the laser source and merge gradually far from the source. The difference at $r=0.005 \mathrm{~cm}$ is $9.5 \%$, with the centered approach underestimating the diffuse reflectance. Because this laser beam is narrow compared with the decay constant of the diffuse reflectance, the convolved diffuse reflectance also merges into the impulse diffuse reflectance far from the laser source. It is also worth noting that the improvement of using the optimized-r positions is the greatest when the beam size is comparable to the size of the grid elements.

Simulated diffuse reflectance and transmittance can be resolved over the exit angle $(\alpha)$ by setting up a grid system in the $\alpha$ domain. Such simulations mimic goniometric measurements of light scattering. ${ }^{12}$ The exit angle is defined as the angle between the light-exiting direction and the normal to the surface of the tissue slab. The simulated diffuse reflectance or transmittance in each $\alpha$-grid element is averaged over the solid angle spanned by the $\alpha$-grid element. The averaged quantity $Y(\alpha)$ in the $n$ th-grid element, which can be either diffuse reflectance or diffuse transmittance, can be expressed as

$$
\begin{aligned}
\langle Y(\alpha)\rangle= & \int_{\alpha_{n}-\Delta \alpha_{n} / 2}^{\alpha_{n}+\Delta \alpha_{n} / 2} Y(\alpha) 2 \pi \sin (\alpha) d \alpha / \\
& \int_{\alpha_{n}-\Delta \alpha_{n} / 2}^{\alpha_{n}+\Delta \alpha_{n} / 2} 2 \pi \sin (\alpha) d \alpha
\end{aligned}
$$

where $\alpha_{n}$ and $\Delta \alpha_{n}$ are the center and width of the grid element, respectively, and the denominator gives the solid angle of the grid element. Since the $\alpha$ grid may not be homogeneous, we use $\Delta \alpha_{n}$ instead of $\Delta \alpha$ for the width of grid element $n$.

Again, if $Y(\alpha)$ in each grid element is differentiable and approximated linearly, we can similarly prove that there exits a best point $\alpha_{b}$ to satisfy (proof not shown)

$$
\langle Y(\alpha)\rangle=Y\left(\alpha_{b}\right),
$$

where

$$
\alpha_{b}=\alpha_{n}+\cot \left(\alpha_{n}\right)\left[1-\frac{\Delta \alpha_{n}}{2} \cot \left(\frac{\Delta \alpha_{n}}{2}\right)\right]
$$

However, the complexity of Eq. (14) will affect its practical value.

For physical quantities, such as light fluence inside the tissue, that can be resolved over $z$ or $t$, it can be easily shown that the best points for the $z$ - and $t$-grid elements are the centers of each grid element (proof not shown).

In conclusion, the simulated physical quantities in each grid element in Monte Carlo modeling should be assigned to the positions that are centered in the $z$ or $t$ domains but not centered in the $r$ or $\alpha$ domains. The error of the estimated physical quantities at $r=0, Y(r=0)$, using the centered- $r$ positions can be up to $14.3 \%$. Using the optimized positions, one can use coarser grids to achieve the same accuracy, yet a smaller statistical variation in Monte Carlo modeling. 
We thank L. Eppich for proofreading the manuscript. This research is supported in part by Navy Medical FEL Program (N00014-91-J-1354), Air Force Office of Scientific Research (F49620-93-1-0298DEF), Department of Energy (DE-FG0591ER61226), and National Institutes of Health (R29. HL45045).

'B. C. Wilson and G. Adam, "A Monte Carlo model for the absorption and flux distributions of light in tissue," Med. Phys. 10, 824-830 (1983).

'S. A. Prahl, M. Keijzer, S. L. Jacques, and A. J. Welch, "A Monte Carlo model of light propagation in tissue," Proc. Soc. Photo-Opt. Instrum. Eng. Inst. Ser. 5, 102-111 (1989) [note the typographical error in Eq. (10), in which the denominator should be $\left.1-g_{0}+2 g_{0} \xi\right]$.

${ }^{3}$ M. Keijzer, S. L. Jacques, S. A. Prahl, and A. J. Welch, "Light distributions in artery tissue: Monte Carlo simulations for finite-diameter laser beams," Lasers Surg. Med. 9, 148-154 (1989).

${ }^{4}$ S. T. Flock, B. C. Wilson, D. R. Wyman, and M. S. Patterson, "Monte Carlo modeling of light propagation in highly scattering tissues. I. Model predictions and comparison with diffusion theory," IEEE Trans. Biomed. Eng. 36, 1162-1168 (1989).

${ }^{5}$ S. T. Flock, B. C. Wilson, and M. S. Patterson, "Monte Carlo modeling of light propagation in highly scattering tissues. I. Comparison with measurements in phantoms," IEEE Trans. Biomed. Eng. 36, 1169-1173 (1989).

"M. Keijzer, J. W. Pickering, and M. J. C. van Gemert, "Laser beam diameter for port wine stain treatment," Lasers Surg. Med. 11, 601-605 (1991).

${ }^{7}$ S. L. Jacques and L.-H. Wang, "Monte Carlo modeling of light transport in tissues," in Optical Thermal Response of Laser Irradiated Tissue, edited by A. J. Welch and M. J. C. van Gemert (Plenum, New York, in press).

${ }^{8} \mathrm{~S}$. L. Jacques, "Time resolved propagation of ultrashort laser pulses within turbid tissues," Appl. Opt. 28, 2223-2229 (1989).

"S. L. Jacques, "Time-resolved reflectance spectroscopy in turbid tissues," IEEE Trans. Biomed. Eng. 36, 1155-1161 (1989).

${ }^{10}$ L.-H. Wang and S. L. Jacques, "Hybrid model of Monte Carlo simulation and diffusion theory for light reflectance by turbid media," J. Opt. Soc. Am. A 10, 1746-1752 (1993).

${ }^{11}$ L.-H. Wang and S. L. Jacques, "Animated simulation of light transport in tissues," Proc. Soc. Photo-Opt. Instrum. Eng. 2134A (in press).

${ }^{12}$ S. L. Jacques, C. A. Alter, and S. A. Prahl, "Angular dependence of HeNe laser light scattering by human dermis," Lasers Life Sci. 1, 309-333 (1987). 